\title{
Betriebsräte und betriebliche Weiterbildung
}

Für viele Betriebsräte ist die Mitbestimmung bei der betrieblichen Weiterbildung nur ein marginales Aktivitätsfeld. In qualitativen Studien wird ihr Engagement auf diesem Gebiet als eher zurückhaltend beschrieben. Dennoch deutet eine Reihe ökonometrischer Untersuchungen darauf hin, dass allein die Existenz eines Betriebsrats einen positiven Einfluss auf die betrieblichen Weiterbildungsaktivitäten ausübt. In diesem Beitrag wird untersucht, inwieweit theoretische Konzepte zur betrieblichen Mitbestimmung Hinweise zur Auflösung dieser widersprüchlichen Befunde bieten. Schließlich werden mögliche Erklärungsansätze für diese scheinbare Diskrepanz vorgestellt. ${ }^{\bullet}$

KLAUS BERGER

\section{Betriebliche Weiterbildung als Handlungsfeld von Betriebsräten}

Das deutsche Berufsbildungssystem ist an der Nahtstelle von Bildungs- und Wirtschaftssystem angesiedelt. Der Betrieb ist der zentrale Lernort. Berufsbildungspolitik vollzieht sich somit auch im Spannungsverhältnis der industriellen Beziehungen. Akteure dieser industriellen Beziehungen sind Arbeitgeberorganisationen und Gewerkschaften auf der überbetrieblichen Ebene; im Betrieb vor Ort das Management und der Betriebsrat.

Auf betrieblicher Ebene wird davon ausgegangen, dass die Interessen von Arbeitgebern und Arbeitnehmern im Bereich der beruflichen Weiterbildung weniger kontrovers sind als bei den traditionellen Betriebsratsthemen wie z. B. die Sicherheit des Arbeitsplatzes. Beide Betriebsparteien haben ein Interesse an guter Qualifizierung der Beschäftigten. Während die Geschäftsleitung jedoch größeren Wert auf betriebsspezifisch verwertbare Qualifikationen zur Stärkung der Wettbewerbsfähigkeit des Unternehmens legt, steht auf Arbeitnehmerseite die Marktgängigkeit der zu vermittelnden Qualifikationen - und damit Arbeitsplatzund Einkommenssicherheit - im Vordergrund.

Die Mitbestimmungsmöglichkeiten der Betriebsräte wurden in den letzten beiden Jahrzehnten durch unterschiedliche Entwicklungen beeinflusst. Mit zunehmendem internationalen Wettbewerbsdruck stiegen die Anforderungen an Produkte und Dienstleistungen und damit auch an die betriebliche Weiterbildung. Spätestens in den 1990er Jahren setzten Betriebe bei Rationalisierungsmaßnahmen nicht mehr nur auf technische Erneuerungen, sondern zunehmend auf Maßnahmen der Personal- und Organisationsentwicklung. Traditionelle Weiterbildungsformen und damit die mitbestimmungsrelevanten Bildungsmaßnahmen schienen einen Bedeutungsverlust zu erleiden. Hingegen gewannen arbeitsintegrierte Lernformen an Bedeutung und stellten die Betriebsräte vor veränderte Anforderungen. Die zunehmende Betonung der individuellen Eigenverantwortung für die berufliche Weiterbildung führte dazu, dass Weiterbildung tendenziell zur „Privatsache“ erklärt und hierdurch dem Einflussbereich der betrieblichen Mitbestimmung entzogen wurde.

\section{Gesetzliche Mitbestimmung}

Die betriebliche Mitbestimmung ermöglicht den Betriebsräten Beteiligungsrechte bei sozialen, personellen und in geringem Maße auch bei wirtschaftlichen Angelegenheiten des Betriebes. Bei betrieblichen Bildungsmaßnahmen haben Betriebsräte Mitgestaltungsmöglichkeiten, soweit diese

Ich danke den Gutachtern für ihre wertvollen Hinweise. 
nicht gesetzlich oder tariflich geregelt sind. Mit der Novellierung des Betriebsverfassungsgesetzes im Jahr 2001 räumte der Gesetzgeber den Betriebsräten in betrieblichen Berufsbildungsfragen ein größeres Mitspracherecht ein. Er unterstrich dabei, dass Mitarbeiterqualifizierung ein Instrument zur Beschäftigungssicherung ist.

Im Gegensatz zur Berufsausbildung, die weitgehend gesetzlich und durch die Ausbildungsordnungen reguliert ist, decken gesetzliche Regelungen zur Weiterbildung nur wenige Teilbereiche der beruflichen Weiterbildung ab (vgl. die landespezifischen Bildungsurlaubsgesetze oder die Regelungen nach SGB II und SGB III). Soweit Tarifvereinbarungen zur beruflichen Weiterbildung bestehen, überlassen diese die konkrete Ausgestaltung der getroffenen Regelungen meist den Betriebsparteien. Betriebsräte haben dabei Informations-, Beratungs-, Vorschlags- und Mitbestimmungsrechte bei der Einführung und Durchführung von Maßnahmen, die der Qualifizierung der Beschäftigten dienen (vgl. insbesondere $\$ \$ 92,96$ bis 98 BetrVG). Diese beziehen sich auf alle Maßnahmen zur Feststellung des Qualifizierungsbedarfs, der Personalbedarfsplanung und -entwicklung, auf die Teilnehmer- und Ausbilderauswahl sowie auf die inhaltliche Gestaltung von Bildungsmaßnahmen. Damit ermöglichen die Mitwirkungsrechte den Betriebsräten auch präventive Handlungsstrategien.

\section{Empirische Befunde zur Rolle von Betriebsräten und betrieblicher Weiterbildung}

Die Wirksamkeit der betrieblichen Mitbestimmung im Bereich der betrieblichen Weiterbildung wurde in einer Reihe von Studien untersucht. Ökonometrische Studien stützen sich meist auf eine Datenbasis (z. B. das IAB-Betriebspanel), die nicht unbedingt mit dem Ziel erhoben wurde, die betriebliche Mitbestimmung zu untersuchen. Der Betriebsratseinfluss auf die betriebliche Weiterbildung wird dabei mittels einer Dummy-Variable zum Vorhandensein eines Betriebsrats und weiterer Kontrollvariablen wie z. B. Betriebsgröße, Branche anhand multivariater Regressionsmodelle geschätzt. Während diese quantitativen Studien keine Hinweise auf das Betriebsratshandeln selbst erlauben, untersuchten Fallstudien in den zurückliegenden Jahrzehnten Betriebsratsaktivitäten und Problemverständnis der Interessenvertretungen im Bereich der betrieblichen Weiterbildung. Die Befunde tragen jedoch nicht unbedingt zur Klärung des statistisch ermittelten Betriebsratseinflusses auf die betriebliche Weiterbildung bei.

Betriebsräte nehmen ihre Aufgabe auf dem Gebiet der Weiterbildung oft nur reaktiv war, indem sie sich für die Interessen einzelner Beschäftigter einsetzen. Um ihre Beteiligungsrechte im Sinne einer strategischen Weiterbildungs- politik auszuschöpfen, fehlen ihnen oft die erforderlichen Ressourcen (vgl. z. B. Bahnmüller/Fischbach 2006). Gestützt wird diese Einschätzung durch eine Reihe qualitativer Studien, die sich seit den 1970er Jahren mit beruflicher Weiterbildung als Handlungsfeld der betrieblichen Mitbestimmung befassen und hier meist ein eher begrenztes Engagement der Betriebsräte feststellten (z. B. Maase et al. 1975; Seusing/Walden 1991; Iller 1993; Zeuner 1997). Die Diskrepanz zwischen dem durchaus vorhandenen Problembewusstsein der Betriebsräte hinsichtlich betrieblicher Weiterbildung (vgl. z. B. Lenz/Voß 2009) und der geringen Ausschöpfung ihrer Mitwirkungsrechte wird auf unterschiedliche Gründe zurückgeführt. Breisig (1997, S. 60) verweist auf ein ,ganzes, Set' von Einzelaspekten, die zu der Zurückhaltung vieler Interessenvertretungen führen“. Bahnmüller/Fischbach (2006, S. 115ff.) identifizierten vier Faktoren, die den Betriebsräten den Umgang mit der betrieblichen Weiterbildung erschweren. Hierzu gehören ein „mangelndes Interesse“ der Beschäftigten, aber auch innerhalb des Betriebsratsgremiums, „mangelnde Kenntnisse und Informationen über die betriebliche Situation“, „fehlende externe Unterstützung“, „fehlende fachliche und rechtliche Kenntnisse“ (ebd., S. 115). Aber auch „Unklarheit über die Ziele, mangelnde Durchsetzungschancen und die Breite des Themas" bereiten den Betriebsräten Probleme (ebd., S 117).

Während diese Studien unterschiedliche Faktoren auflisten, die die Betriebsratsarbeit auf dem Gebiet der Weiterbildung erschweren und damit das geringe Engagement der Interessenvertretungen begründen, gehen Backes-Gellner et al. (1997, S. 334ff.) von einem theoriegeleiteten Erklärungsansatz aus. Die Gründe für das geringe Betriebsratsengagement liegen demnach weniger in den genannten externen Faktoren als vielmehr im Verhandlungsgegenstand selbst. Da die Berufsbildung in den betrieblichen Sozialbeziehungen ein relativ konfliktfreies Handlungsfeld darstelle, gäbe es für Betriebsräte kaum Anlass, hier aktiv zu werden. In Anlehnung an Becker (1962) unterscheiden die Autoren zwischen „general marketable skills“, die eher im Interesse der Beschäftigten liegen und „firm-specific skills“, die im Interesse des Arbeitgebers sind. Ähnlich wie später Lazear (2003) stellen sie allerdings die praktische Relevanz dieser theoretischen Unterscheidung infrage. Sie verweisen dabei auf empirische Befunde, nach denen in der betrieblichen Weiterbildungspraxis nie ausschließlich ,firm-specific skills“, sondern immer auch „general marketable skills“ vermittelt werden. Betriebliche Weiterbildung nutzt damit nicht nur den betriebsspezifischen Verwertungsinteressen des Arbeitgebers, sondern vermittelt auch „general skills“, die die Arbeitsmarktmobilität der Beschäftigten erhöhen können. In seinem „Skill-Weights Approach“ spricht Lazear daher nur noch von betriebsspezifisch gewichteten Kombinationen verschiedener „general skills“, deren Verwertungsmöglichkeiten sich im Einzelnen bei freiwilligen oder erzwungenen Betriebswechseln je nach Arbeitsmarktlage erheblich verändern können.

Sowohl im Ansatz von Lazear als auch in der Betrachtung von Backes-Gellner et al. überwiegt bei der be- 
trieblichen Weiterbildungspraxis der gemeinsame Nutzen für Betriebe und Beschäftigte. Gestützt wird diese Annahme durch die Überlegung, dass Betriebsräte nicht nur die Vermittlung von „general marketable skills“ unterstützen, sondern auch an der Vermittlung von "firm-specific skills" interessiert sind, wenn hierdurch die Wettbewerbsfähigkeit des eigenen Unternehmens im Interesse der Arbeitsplatzsicherheit gestärkt wird (Backes-Gellner et al. 1997, S. 334). Unter diesen Voraussetzungen gehen die Autoren davon aus, dass Betriebsräte sich bei der Wahrnehmung ihrer gesetzlichen Mitbestimmungsrechte in der Berufsbildung auf ihre traditionelle Verteidigungsrolle beschränken und dabei versuchen, Benachteiligungen bei der Teilnehmerauswahl sowie Beschäftigungs- und Einkommensrisiken zu vermeiden.

Backes-Gellner et al. erklären die Befunde aus den qualitativen Studien zwar schlüssig damit, dass Betriebsräte nur deshalb ein geringes Engagement bei der betrieblichen Weiterbildung zeigen, weil sich ihre Interessen auf diesem Gebiet meist kaum von denen der Geschäftsleitung unterscheiden. Nicht erklären können sie jedoch die Befunde von ökonometrischen Studien zum positiven Betriebsratseinfluss auf betriebliche Weiterbildungsaktivitäten bzw. auf Produktivitätseffekte von Weiterbildung (z. B. Bellmann/ Ellguth 2006; Leber 2009; Stegmaier 2010). Hiernach wäre eher eine aktive Weiterbildungspolitik der Betriebsräte im Rahmen der bestehenden Mitwirkungsrechte zu erwarten. Da die Befunde qualitativer Studien dies jedoch nicht bestätigen, muss nach anderen Erklärungen für den positiven Effekt von Betriebsräten auf das betriebliche Weiterbildungsangebot und die Weiterbildungsbeteiligung gesucht werden. Auch Bellmann und Ellguth (2006) konnten nicht nachweisen, dass Betriebsräte ihre seit 2001 erweiterten Mitbestimmungsmöglichkeiten auch wirklich nutzen.

\section{Theoretische Erklärungsansätze zur betrieblichen Mitbestimmung}

Im Folgenden soll der Frage nachgegangen werden, ob theoretische Konzepte zur betrieblichen Mitbestimmung Aufschluss darüber geben, warum trotz der nur geringen Betriebsratsaktivitäten signifikante Betriebsratseffekte im Bereich der betrieblichen Weiterbildung festzustellen sind. Hierzu werden Theorieansätze zur Wirksamkeit und zum Nutzen von betrieblichen Interessenvertretungen sowie Konzepte herangezogen, die die betriebliche Mitbestimmung als sozialen Prozess beschreiben.

\subsection{Institutionenökonomische Ansätze zum Nutzen betrieblicher Mitbestimmung}

Die Institutionenökonomie bietet unterschiedliche Argumente, die aus Unternehmenssicht sowohl für als auch gegen die betriebliche Mitbestimmung sprechen (Dilger
2002). Danach trägt der Betriebsrat dazu bei, die Informationsasymmetrie zwischen Management und Beschäftigten auszugleichen. Das Vertrauen der Beschäftigten in Leitungsentscheidungen werde gesteigert, weil der Betriebsrat sich die Anliegen des Managements anhört und die Beschäftigten davon ausgehen können, dass er die Glaubwürdigkeit der Entscheidungsgrundlagen prüft (Ear-Ansatz).

Während mit dem Transaktionskostenansatz begründet wird, dass kollektive Interessenvertretungen zur Kostenminimierung bei innerbetrieblichen Abstimmungsprozessen zwischen Geschäftsleitung und einzelnen Mitarbeitern beitragen, argumentiert der Exit-Voice-Ansatz mit der Motivation der Beschäftigten. So werde die Arbeitszufriedenheit gesteigert und das Risiko der (inneren) Kündigung (Exit-Option) verringert, wenn die Beschäftigten über einen Betriebsrat die Möglichkeit erhalten, ihre Interessen gegenüber der Geschäftsleitung zu vertreten. Ferner habe betriebliche Mitbestimmung auch einen Eigenwert, da Beschäftigte mit Partizipationsmöglichkeiten aus ihrem Arbeitsverhältnis einen größeren Nutzen ziehen und dadurch motivierter und produktiver sind (Eigenwert-Ansatz). Die daraus folgende geringere Personalfluktuation erhöhe einerseits den Anreiz für Betriebe, in Weiterbildung zu investieren, andererseits bieten stabile Beschäftigungsverhältnisse für die Beschäftigten selbst wiederum Weiterbildungsanreize. Die Existenz eines Betriebsrats hätte somit zumindest mittelbar Auswirkungen auf die betrieblichen Weiterbildungsaktivitäten und auf die Weiterbildungsbereitschaft der Beschäftigten. Der Vorteil dieses Erklärungsansatzes liegt darin, dass er keine besonderen Betriebsratsaktivitäten im Bereich der betrieblichen Weiterbildung voraussetzt, sondern davon ausgeht, dass Betriebsräte ihrer gesetzlichen Aufgabe gerecht werden und dadurch mittelbar zu einem weiterbildungsfreundlichen Betriebsklima beitragen. Bisherige Studien zeigen jedoch, dass diese Voraussetzung nicht immer gegeben ist und die Effizienz von Betriebsräten sehr unterschiedlich sein kann (Kotthoff 1994).

\subsection{Arenenkonzept}

Nicht ökonomische Effizienzüberlegungen zur betrieblichen Mitbestimmung sind der Ausgangspunkt des Arenenkonzepts von Müller-Jentsch (1999), sondern es geht um den Interessengegensatz von Arbeit und Kapital. Im Laufe der historischen Entwicklung dieses Interessenkonflikts haben sich Institutionen der geregelten Konfliktaustragung zwischen Arbeitgebern und Arbeitnehmern herausgebildet, die arbeitsteilig auf zwei Ebenen ausgetragen werden. Institutionelle Grundlage ist hier zum einen die Tarifautonomie mit dem Streikrecht der Gewerkschaften und zum anderen die Betriebsverfassung mit den Beteiligungsrechten der Betriebsräte. Die tarifliche und betriebliche Ebene werden auch als Arenen der Arbeitsbeziehungen bezeichnet. Eine Arena ist dabei der ,Ort' geregelter Konfliktaustragung und institutionalisierter Problemlösung, [...], auf dem die 
jeweiligen Akteure nicht nur ihre widerstreitenden Interessen durchsetzen, sondern auch die prozeduralen Rahmenbedingungen zu verändern trachten." (ebd., S. 233).

Im Folgenden wird gezeigt, dass das Austragen dieses Interessenkonflikts auf einer Machtbeziehung beruht und durch „Informalität und Historizität“ geprägt ist (Bosch 1997, S. 44ff.). Ferner ist davon auszugehen, dass es bestimmter Ressourcen bedarf, damit eine Belegschaft die Möglichkeiten der geregelten Konfliktaustragung zur Durchsetzung ihrer Interessen auch nutzen kann.

\subsection{Interessen- und machttheoretische Sicht}

Das Aushandeln von Interessen zwischen Management und Beschäftigten bzw. Betriebsrat wird durch die Kategorie der Macht bestimmt (Bosch 1997). Jürgens (1984) unterscheidet hierbei zwischen „Primärmacht“ und „Sekundärmacht“. Unter „Primärmacht" versteht er, „die originär aus der Art der Abhängigkeitsbeziehungen zwischen den sozialen Parteien im Betrieb erwachsenen Machtpositionen für einzelne Beschäftigte bzw. Beschäftigtengruppen“.

„Sekundärmacht“ beruht „,auf bereits kollektiv erkämpften bzw. staatlich gesetzten Regelungen und Institutionen“" (ebd., S. 61). Im Rückgriff auf Crozier und Friedberg (1979, hier S. 41) ist Macht „,in dem Freiraum angesiedelt, über den jeder der in eine Machtbeziehung eingetretenen Gegenspieler verfügt, das heißt, in seiner mehr oder weniger großen Möglichkeit, das zu verweigern, was der andere von ihm verlangt“. Macht wird nicht als ein Attribut der Akteure, sondern als Beziehung definiert. Im Verhältnis von Arbeitgebern und Arbeitnehmern handelt es sich um eine asymmetrische Machtbeziehung, „die aus der ökonomischen Grundkonstellation“ (Braun 2002, S. 87) von Kapital und Arbeit herrührt und im historischen Prozess dazu geführt hat, dass die Primärmacht durch den oft erstrittenen Aufbau kollektiver Interessenvertretungen ,ersetzt, ergänzt, überlagert" wurde (Jürgens 1984, S. 64).

Damit wurde die Primärmacht der Beschäftigten jedoch nicht bedeutungslos. Indem Beschäftigte über Weiterbildung ihr Qualifikationsniveau steigern, verbessern sie - je nach Arbeitsmarktsituation - auch ihre Exit-Optionen und damit ihre individuelle Verhandlungsmacht gegenüber dem Arbeitgeber. Schließlich bedarf es auch der Primärmacht der Beschäftigten, um überhaupt einen Betriebsrat zu installieren. Der ressourcentheoretische Ansatz von Pongratz und Trinczek (2007) gibt einen Hinweis darauf, welche Bedeutung die Qualifikation der Belegschaft eines Betriebes hierbei haben kann.

\subsection{Ressourcentheoretische Sicht}

Ausgehend von der ungleichgewichtigen (ökonomischen) Ressourcenausstattung zwischen Arbeitgebern und Arbeitnehmern entwickeln Pongratz und Trinczek eine ressourcentheoretische Sicht der industriellen Beziehungen. Sie greifen dabei auf Bourdieus (1992) Kategorien des ökonomischen, sozialen und kulturellen Kapitals zurück. Ökonomisches Kapital ist dabei z. B. im Eigentumsrecht institutionell verankert, während kulturelles Kapital, wie z. B. Bildungsabschlüsse, unmittelbar mit der Person als Träger verbunden ist. Soziales Kapital beruht hingegen auf der Zugehörigkeit zu einer bestimmten Gruppe. Die von Bourdieu untersuchten Transformationsbeziehungen zwischen den Kapitalformen sehen die Autoren auch bei den industriellen Beziehungen als gegeben an. So gehe es ressourcenanalytisch betrachtet bei Erwerbsarbeit bzw. bei den ihr zugrunde liegenden Arbeitsverträgen vereinfacht um die Transformation kulturellen Kapitals (vorrangig in Form von Berufskompetenzen) in ökonomisches Kapital (Lohn, Unternehmensgewinn). „In die kollektive Interessenvertretung investieren die Beschäftigten hingegen vorwiegend soziales Kapital (in Form von Mitgliedschaft, Unterstützung, Identifikation), um die mit dem Einsatz des kulturellen Kapitals (ihrer Arbeitskraft) erworbenen individuellen Erwerbschancen durch Interessenkoalition zu sichern“ (Pongratz/Trinczek 2007). Ob dies gelingt, dürfte dabei auch von der Personalstruktur eines Unternehmens abhängen. So ist zu vermuten, dass das Arbeitsplatzrisiko von Beschäftigten in weitgehend gering qualifizierten Belegschaften eher steigt, sobald diese in innerbetrieblichen Konfliktsituationen versuchen, ihre gemeinsamen Interessen kollektiv zu vertreten. Erst bei steigendem Risiko des Arbeitgebers, am Arbeitsmarkt keine seinen Qualifikationsanforderungen entsprechenden Fachkräfte zu finden, verfügen Belegschaften über ein ausreichendes kulturelles Kapital, um sich für die Gründung eines Betriebsrats einsetzen zu können, ohne gleich eine Kündigung zu riskieren. D. h., es bedarf eines gewissen kulturellen Kapitals, damit Beschäftigte in eine kollektive Interessenvertretung investieren können. In der Regel trifft dies auf Belegschaften zu, die einen hohen Facharbeiteranteil haben und über einen ausreichenden gewerkschaftlichen Organisationsgrad verfügen. Gehen die Qualifikationen der Beschäftigten jedoch deutlich darüber hinaus, vertrauen sie eher auf die ihnen hieraus erwachsende Primärmacht und ziehen eine individuelle Interessenvertretung der kollektiven Variante vor.

Auch der Betriebsrat benötigt ein entsprechendes kulturelles Kapital (in Form von Wissen, Kenntnissen und Verhandlungsgeschick), damit er seine Mitbestimmungsrechte bei betrieblichen Berufsbildungsmaßnahmen nutzen kann, um so auf günstige Rahmenbedingungen für die Generierung und Akkumulation kulturellen Kapitals der Beschäftigten hinzuwirken. Dieses dürfte er insbesondere dann einsetzen, wenn es sich bei Erfolg wieder in soziales Kapital (z. B. Akzeptanz, Unterstützung, Vertretungswirksamkeit) für weitere Aktivitäten des Betriebsrats auszahlt. Umgekehrt bedeutet dies, dass Betriebsräte betriebliche Politikfelder, in denen Beschäftigte ihre Interessen z. B. an betrieblicher Weiterbildung direkt mit ihren Vorgesetzten aushandeln, dann eher nachrangig behandeln, wenn sie nicht erwarten können, dass ihre Aktivitäten in diesen Feldern auch von der Belegschaft positiv aufgenommen werden. Das ist beispielsweise dann der Fall, wenn die 
Beschäftigten aufgrund ihrer Qualifikationen über ein so hohes kulturelles Kapital und damit eine entsprechende Primärmacht verfügen, dass sie Ihre Interessen z. B. im Bereich der Weiterbildung individuell mit Ihren Vorgesetzten selbst aushandeln. Nicht zuletzt kann davon ausgegangen werden, dass Arbeitgeber, die weitgehend auf qualifizierte Fachkräfte angewiesen sind, ein grundsätzliches Interesse haben, dieses kulturelle Kapital in Form von betriebsspezifischen Qualifikationen zu sichern und weiterzuentwickeln.

\subsection{Interaktionskulturelle Sicht}

Das Betriebsverfassungsgesetz gibt zwar den rechtlichen Rahmen für die kollektive Interessenvertretung vor, für die Interaktionsbeziehungen zwischen Management und Betriebsrat ist jedoch die informelle und situationsspezifische Ausgestaltung des gesetzlichen Handlungsrahmens entscheidend (Bosch 1997, S. 44ff.). Die Ergebnisse dieses Prozesses können im Sinne des „negotiated-order“-Ansatzes auch als ausgehandelte Ordnung begriffen werden. Sie verfestigen sich zu betriebshistorisch gewachsenen Strukturen, die es ermöglichen, dass in vergleichbaren Situationen Interpretationen und Handlungsweisen, solange sie nicht zu negativen Erfahrungen führen, aktualisiert und wiederholt werden. Entsprechend sind auch die betrieblichen Interaktionsbeziehungen der Geschäftsleitung zur Belegschaft durch Informalität und Historizität geprägt. Fragen der individuellen Personalentwicklung und Weiterbildung werden z. B. eher mit den unmittelbaren Vorgesetzten ausgehandelt, ohne dass der Betriebsrat hiervon Kenntnis bekommt. Solange dies den Beschäftigten funktional erscheint, wird die Inanspruchnahme einer kollektiven Interessenvertretung nicht nur als nicht erforderlich, sondern möglicherweise eher als konfliktfördernd wahrgenommen. Unter machttheoretischen Gesichtspunkten findet die Aushandlung von Weiterbildungsmöglichkeiten auf der Grundlage der jeweiligen „Primärmacht“ der Mitarbeiter statt (Jürgens 1984). Dies kann zur Folge haben, dass eine dezentrale und informelle Regelung betrieblicher Weiterbildung den Betriebsrat von Informationen zum dezentralisierten Weiterbildungsgeschehen abschneidet und ihm damit eine wichtige Grundlage für sein Engagement auf diesem Gebiet entzogen wird.

\section{Passive Betriebsräte mit positiven Weiterbildungseffekten - kein Widerspruch?}

Die im vorangegangenen Abschnitt dargestellten Theorieansätze beschreiben die betriebliche Mitbestimmung zum einen unter ökonomischen Nutzenaspekten und zum anderen lenken sie den Blick auf den sozialen Prozess der Aus- tauschbeziehungen zwischen Management und Betriebsrat. Sie zeigen, dass eine institutionalisierte Interessenvertretung die Betriebsbindung der Beschäftigten und damit auch einen Weiterbildungsanreiz für Betrieb und Belegschaft erhöhen kann. Voraussetzung hierfür ist jedoch, dass ein Betriebsrat gegründet wird. Damit wäre ein Hinweis gegeben, dass allein die Existenz eines Betriebsrats betriebliche Weiterbildungsaktivitäten fördert. Dies setzt zwar nicht voraus, dass sich der Betriebsrat für Weiterbildung engagiert, jedoch müssen die Beschäftigten ihn generell als effektive Interessenvertretung wahrnehmen können.

Auf einen mittelbaren Zusammenhang zwischen der Existenz eines Betriebsrats und betrieblichen Weiterbildungsaktivitäten weist auch der ressourcentheoretische Ansatz hin. So ist davon auszugehen, dass Betriebsbelegschaften, die mehrheitlich aus qualifizierten Fachkräften bestehen, über günstigere Voraussetzungen für die Betriebsratsgründung verfügen als Belegschaften mit gering qualifizierten Arbeitskräften. Gleichzeitig dürfte den Arbeitgebern an einer Betriebsbindung und der Weiterbildung ihrer qualifizierten Fachkräfte grundsätzlich gelegen sein, wenn die Deckung des Fachkräftebedarfs über den Arbeitsmarkt aufwendiger und mit größeren Fehlentscheidungsrisiken behaftet ist. Auch in diesem Fall ist kein unmittelbares Betriebsratsengagement im Bereich der betrieblichen Weiterbildung erforderlich, vielmehr erhöht die Qualifikationsstruktur der Betriebsbelegschaft sowohl die Chancen für Betriebsratsgründungen als auch für betriebliche Weiterbildungsaktivitäten.

Ein solches Weiterbildungsengagement wird von Betriebsräten möglicherweise auch nicht für nötig gehalten, da es gerade die qualifizierten Fachkräfte sind, die vorrangig ihre Primärmacht nutzen, um ihre Weiterbildungsinteressen unmittelbar im Verhältnis von Beschäftigten zu Vorgesetzten auszuhandeln, ohne den Betriebsrat in Anspruch zu nehmen. In dieser Konstellation kann dem Betriebsrat ein eigenes Engagement für die Weiterbildungsinteressen der Belegschaft auch angesichts knapper eigener Ressourcen als wenig gewinnbringend erscheinen.

Wird vorausgesetzt, dass Arbeitgeber und Betriebsrat an der Qualifizierung der Beschäftigten weitgehend übereinstimmende Interessen haben, wäre schließlich denkbar, dass die betriebsspezifisch gewachsenen Interaktionsstrukturen und wechselseitigen Erfahrungen in der geregelten Konfliktaustragung zwischen den Betriebsparteien für sich genommen bereits einen Anreiz darstellen, eine bestimmte Intensität der betrieblichen Weiterbildungspraxis aufrechtzuerhalten, nicht zuletzt um eine kooperative Haltung des Betriebsrats auch in anderen Politikfeldern zu fördern. Auf der Grundlage dieser Überlegungen werden im folgendem Abschnitt Erklärungsansätze vorgestellt, die einen Zusammenhang zwischen der Existenz eines Betriebsrats und höheren betrieblichen Weiterbildungsaktivitäten zulassen, ohne dass dies gesteigerte Betriebsratsaktivitäten auf diesem Gebiet voraussetzt. Hierbei soll nicht ausgeschlossen werden, dass weitere Erklärungen für dieses Phänomen möglich sind. 


\subsection{Mitbestimmungsstrukturen sichern betriebliche Bildungsaktivitäten}

Wenn nicht die unmittelbaren Aktivitäten des Betriebsrats auf dem Gebiet der Weiterbildung für den fördernden Einfluss des Betriebsrats auf das betriebliche Weiterbildungsgeschehen verantwortlich sein können, stellt sich die Frage, inwieweit strukturelle Aspekte der betrieblichen Mitbestimmung hierzu beitragen.

Die Förderung von betrieblicher Weiterbildung wie auch der Personalentwicklung insgesamt lässt sich als originäre Forderung von Gewerkschaften und Betriebsräten und damit durchaus als ein sinnstiftendes Element beider Institutionen verstehen. Für den Betriebsrat wird dieser sinnstiftende Aspekt dadurch verstärkt, dass die Förderung von betrieblicher Weiterbildung zu seinem gesetzlichen Aufgabenkatalog gehört. Die Annahme von Backes-Gellner et al. (1997), dass der Betriebsrat im Grundsatz ähnlich ausgerichtete Interessen wie die Geschäftsleitung verfolgt und deshalb, wie die Befunde vieler qualitativer Studien zeigen, im Handlungsfeld der betrieblichen Weiterbildung auf eigene Aktivitäten eher verzichtet, lässt erwarten, dass die Weiterbildungsaktivitäten von mitbestimmten Betrieben und betriebsratslosen Betrieben sich nicht unterscheiden. Die ökonometrischen Studien kommen jedoch zu gegenteiligen Befunden.

Ein Grund mag darin liegen, dass Betriebsrat und Geschäftsleitungen zwar im Bereich der betrieblichen Weiterbildung im Grundsatz ähnlich ausgerichtete Interessen haben, diese aber auf unterschiedlichen Handlungslogiken beruhen. Während betriebliche Bildungsaktivitäten für den Betrieb „im Rahmen ökonomischer Verwertung von Arbeitskraft" stattfinden und notwendigerweise darauf ausgerichtet sind (Geißler 1990, S. 200), steht für Betriebsräte das Beschäftigteninteresse an Arbeitsplatzsicherheit und beruflicher Entwicklung im Vordergrund. Dass Betriebe mit Betriebsrat weiterbildungsaktiver als betriebsratslose Betriebe sind, deutet somit darauf hin, dass mitbestimmte Betriebe den Betriebsrat und seine Anforderungen an die betriebliche Weiterbildung bei strategischen Entscheidungen zu betrieblicher Bildung in ihr ökonomisches Kalkül einbeziehen. Selbst wenn ein Absenken von Weiterbildungsaktivitäten auf das Niveau betriebsratsloser Betriebe ökonomisch vertretbar oder sogar geboten erscheint, müssen doch in mitbestimmten Betrieben ein möglicher Widerstand und daraus resultierende Beeinträchtigungen der Kooperationsbereitschaft des Betriebsrats berücksichtigt werden.

Genauso wie betriebshistorisch gewachsene Interaktionsstrukturen dazu führen, dass in vergleichbaren Situationen Interpretationen und Handlungsweisen, solange sie nicht zu negativen Erfahrungen führen, aktualisiert und wiederholt werden (Bosch 1997, S. 46), wird auch das Wissen um die Interessen und die Handlungsoptionen des Betriebsrates bereits im Vorfeld in die strategischen Überlegungen des Managements einfließen. Das Interesse des Managements, sich die kooperative Haltung des Betriebsrats zu bewahren, kann somit zur Aufrechterhaltung von betrieblichen Weiterbildungsaktivitäten beitragen. Dies setzt zwar einen in traditionellen Politikfeldern aktiven Betriebsrat voraus, Träger einer aktiven betrieblichen Weiterbildungspolitik bleibt jedoch in dieser Konstellation in erster Linie das Management und nicht der Betriebsrat.

Denkbar ist hierbei auch eine Arbeitsteilung zwischen Personalentwicklungsabteilung und Betriebsrat. Breisig (1997, S. 55) verweist darauf, dass „, das Management ‘ ja kein homogener Block mit gleichgerichteten Interessen ist.“ Aufgrund der „fehlenden Verankerung in den unternehmenspolitisch wichtigen Netzwerken" werde die strategische Bedeutung von Personalentwicklungsabteilungen für den Unternehmenserfolg ,trotz anderweitiger Bekundungen selbst im Großunternehmen" immer noch als nachrangig betrachtet (ebd., S. 56). Daraus kann sich eine Interessenlage ergeben, die Personalentwicklungsabteilungen zum potenziellen Bündnispartner von Betriebsräten machen. Diese Bündniskonstellation kann betriebliche Weiterbildungsaktivitäten fördern, ohne dass hierzu ein ausdrücklich aktives Betriebsratsengagement erforderlich ist. Eine solche Arbeitsteilung könnte beinhalten, dass der Betriebsrat Initiativen der Personalentwicklungsabteilung zwar mitträgt, sich selbst jedoch im Bereich der betrieblichen Weiterbildung auf eine individuelle Konfliktberatung und formalisierte gesetzliche Mitwirkungsroutinen beschränkt. Trotzdem erfahren die Personalentwicklungsabteilung und ihre Aktivitäten allein durch die Signalwirkung der - wenn auch nur passiv mitgetragenen - Zustimmung des Betriebsrats eine innerbetriebliche Aufwertung.

\subsection{Einbindung des Betriebsrats und betriebliche Weiterbildung}

Wie bereits gezeigt, können allein bestimmte Qualifikationsstrukturen in der Belegschaft die Gründung eines Betriebsrats begünstigen bzw. auch erschweren. Qualifizierte Belegschaften verfügen danach über bessere Voraussetzungen, gleichzeitig hat auch der Arbeitgeber ein grundlegendes Interesse, deren Qualifikationen zu erhalten und weiterzuentwickeln.

Die Bedeutung qualifizierter Fachkräfte für den Unternehmenserfolg steht insbesondere bei der Unternehmensstrategie des Human-Resource-Managements (HRM) im Mittelpunkt. Qualifizierte Beschäftigte werden als knappe und schwer ersetzbare Ressource verstanden (Hucker 2008, S. 119ff.). Da mit der Qualifikation der Beschäftigten sich auch deren „Exit-Optionen“ verbessern, gilt Mitarbeiterpartizipation als ein wichtiger Baustein, um die Beschäftigten durch Förderung der Arbeitszufriedenheit und -motivation an das Unternehmen zu binden und um gleichzeitig deren ungenutzte Kompetenzen zu erschließen. Sie ermöglicht die Ermittlung individueller Weiterbildungsbedarfe und erhöht nicht zuletzt die Attraktivität des Unternehmens im Wettbewerb um hoch qualifiziertes Personal (ebd., S. 124ff.).

Weitbrecht und Fischer (1993, S. 202) zeigten, dass bessere Arbeitsbeziehungen zwischen Management und Beschäftigten auch durch kooperative Beziehungen mit dem 
Betriebsrat gefördert werden. Ferner fördern Geschäftsleitungen, die zum Betriebsrat kooperative Beziehungen pflegen, in den Augen der Beschäftigten die Glaubwürdigkeit von Unternehmensentscheidungen (Ear-Ansatz) und tragen somit zur Senkung von Transaktionskosten bei. Es liegt demnach auch im Interesse einer HRM-orientierten Unternehmensführung, innerbetriebliche Sozialbeziehungen nicht nur auf der individuellen, sondern auch auf der kollektiven Ebene zu fördern, zumal Betriebsräte in den Unternehmen mit 250 und mehr Beschäftigten nach wie vor von erheblicher Bedeutung sind. Die Förderung guter Arbeitsbeziehungen auf individueller wie auf kollektiver Ebene durch HRM-Elemente wie Mitarbeiterqualifizierung und Personalentwicklung wären damit Instrumente ein und derselben Managementstrategie. Auch dies würde erklären, dass die Existenz von Betriebsräten und positive betriebliche Bildungsaktivitäten nicht auf entsprechende Betriebsratsaktivitäten zurückgehen müssen, sondern beides letztlich integraler Bestandteil einer HRM-orientierten Unternehmensstrategie sein kann.

\section{Resümee}

Betriebliche Weiterbildung ist für sich genommen oft nur ein nachrangiges Handlungsfeld von Betriebsräten. Dennoch zeigen Betriebe, die einen Betriebsrat haben, im Bereich der Weiterbildung intensivere Bildungsaktivitäten als betriebsratslose Betriebe. In dem Beitrag wurde gezeigt, dass es sich hier um einen nur scheinbaren Widerspruch handelt, der sich bei näherer Betrachtung der theoretischen Erklärungskonzepte zur betrieblichen Mitbestimmung auflösen lässt. Innerbetriebliche Mitbestimmungsstrukturen und intensivere betriebliche Weiterbildungsaktivitäten können somit Bestandteil der gleichen Unternehmensstrategie sein. Die bisherigen Studien zeigen, dass dies ein spezifisches Engagement des Betriebsrats im Bereich der Weiterbildung nicht unbedingt voraussetzt. Damit jedoch auch Arbeitnehmerinteressen bei der Ausgestaltung der betrieblichen Weiterbildungsaktivitäten berücksichtigt werden, dürften dennoch vorausschauende Handlungsstrategien des Betriebsrats erforderlich sein. Ansatzpunkte für ein solches Betriebsratsengagement bestehen darin, dass es im Bereich der betrieblichen Weiterbildung nicht nur Interessengegensätze, sondern eher als in traditionellen Politikfeldern des Betriebsrats auch Interessenüberschneidungen gibt und damit günstigere Voraussetzungen für Kooperationsmöglichkeiten zwischen Management und Betriebsrat vorliegen. Hier ist auszuloten, inwieweit Verantwortliche für Personalentwicklung Bündnispartner einer strategisch angelegten Weiterbildungspolitik des Betriebsrats sein können. Impulse für ein Betriebsratsengagement können dabei auch von Qualifizierungstarifverträgen ausgehen.

\section{LITERATUR}

Backes-Gellner, U./Frick, B. /Sadowski, D. (1997): Codetermination and personnel policies of German firms: the influence of works councils on turnover and further training, in:The International Journal of Human Resource Management 8 (3), S. $328-347$

Bahnmüller, R./Fischbach, S. (2006): Qualifizierung und Tarifvertrag. Befunde aus der Metallindustrie Baden-Württembergs, Hamburg

Bellmann, L./Ellguth, P. (2006): Verbreitung von Betriebsräten und ihr Einfluss auf die betriebliche Weiterbildung, in: Jahrbücher für Nationalökonomie und Statistik 226 (5), S. 487-504
Becker, G. S. (1962): Investment in human capital: A theoretical analysis, in: Journal of Political Economy 70 (1), S. 9-49

Bosch, A. (1997): Vom Interessenkonflikt zur Kultur der Rationalität. Neue Verhandlungsbeziehungen zwischen Management und Betriebsrat, München/Mering Bourdieu, P. (1992 [1983]): Ökonomisches Kapital - Kulturelles Kapital -

Soziales Kapital, in: Bourdieu, P.: Die verborgenen Mechanismen der Macht, Hamburg, S. 49-80

Braun, W. M. (2002): Strategisches Management der industriellen Beziehungen. Zur Empirie und Theorie des Verhältnisses zwischen Management und Betriebsrat, München/Mering

Breisig, T. (1997): Personalentwicklung und Qualifizierung als Handlungsfeld des Betriebsrats. Grundlagen - Maßnahmen - Betriebs- und Tarifvereinbarungen, Baden-Baden

Crozier, M./Friedberg, E. (1979): Macht und Organisation. Die Zwänge kollektiven Handelns, Königstein

Dilger, A. (2002). Ökonomik betrieblicher Mitbestimmung. Die wirtschaftlichen Folgen von Betriebsräten, München/Mehring

Geißler, K. A. (1990): Viel Neues in der betrieblichen Bildungsarbeit und manches Alte auch, in: Herzer, H./Dybowski, G./Bauer, H. G. (Hrsg.): Methoden betrieblicher Weiterbildung, Eschborn, S. 198-222

Hucker, T. (2008): Betriebliche Partizipation und gesellschaftlicher Wandel, München/Mehring

Iller, C. (1993): Interessenvertretung und betriebliche Weiterbildung. Empirische Ergebnisse zu Problemen und Handlungsansätzen von Betriebsräten im Einzelhandel, Bremen

Jürgens, U. (1984): Die Entwicklung von Macht, Herrschaft und Kontrolle im Betrieb als politischer Prozeß - Eine Problemskizze zur Arbeitspolitik, in: Jürgens, U./Naschhold, F. (Hrsg.): Arbeitspolitik, Opladen

Kotthoff, H. (1994): Betriebsräte und Bürgerstatus, München/Mehring

Lazear, E. P. (2003): Firm-Specific Human Capital: A Skill-Weights Approach. National Bureau of Economic Research, NBER Working Papers (9679), Cambridge, MA

Leber, U. (2009): Betriebsgröße, Qualifikationsstruktur und Weiterbildungsbeteiligung. Ergebnisse aus dem IAB-Betriebspanel, in: Behringer, F./Käpplinger, B./Pätzold G. (Hrsg.): Betriebliche Weiterbildung, Stuttgart, S. 149-168

Lenz, K./Voß, A. (2009): Analyse der Praxiserfahrungen zum Qualifizierungstarifvertrag der Metall- und Elektroindustrie NRW, Hans-Böckler-Stiftung, Arbeitspapier (172), Düsseldorf

Maase, M./Sengenberger, W./Weltz, F. (1975): Weiterbildung - Aktionsfeld für den Betriebsrat? Eine Studie über Arbeitnehmerinteressen und betriebliche Bildungspolitik, München

Müller-Jentsch, W. (1999): Berufsbildung - eine Arena der industriellen Beziehungen?, in: Harney, K. (Hrsg.): Beruf und Berufsbildung, Weinheim, S. 233-248 Pongratz, H. J./Trinczek, R. (2007): Industrielle Beziehungen als soziales und kulturelles Kapital. Innovative Bildungs- und Qualifizierungskonzepte von Gewerkschaften und Unternehmen, in: Moldaschl, M. (Hrsg.): Immaterielle Ressourcen. Nachhaltigkeit von Unternehmensführung und Arbeit I, München/ Mering, S.137-172

Seusing, B./Walden, G. (1991): Die Beteiligung von Betriebsräten an der Gestaltung der betrieblichen Weiterbildung, in: Gewerkschaftliche Bildungspolitik (10), S. $232-235$

Stegmaier, J. (2010): Effects of Workplace Representation on Firm-Provided FurtherTraining in Germany, IAB-Discussion Paper, Nürnberg

Weitbrecht, H./Fischer, S. (1993): Human Resource Management und industrielle Beziehungen, in: Müller-Jentsch, W. (Hrsg.): Schriftenreihe Industrielle Beziehungen, München/Mehring, S. 179-208

Zeuner, C. (1997): Betriebliche Weiterbildung - ein neues Politikfeld für Betriebsräte. Ergebnisse aus dem FORCE-Projekt „Einflussmöglichkeiten von Betriebsräten auf die Weiterbildungspolitik ihrer Unternehmen im Rahmen des sozialen Dialogs", Bielefeld

\section{AUTOR}

KLAUS BERGER, Dipl. Päd., ist wissenschaftlicher Mitarbeiter im Bundesinstitut für Berufsbildung (BIBB). Arbeitsbereich: Kosten, Nutzen, Finanzierung der Berufsbildung.

berger@bibb.de 